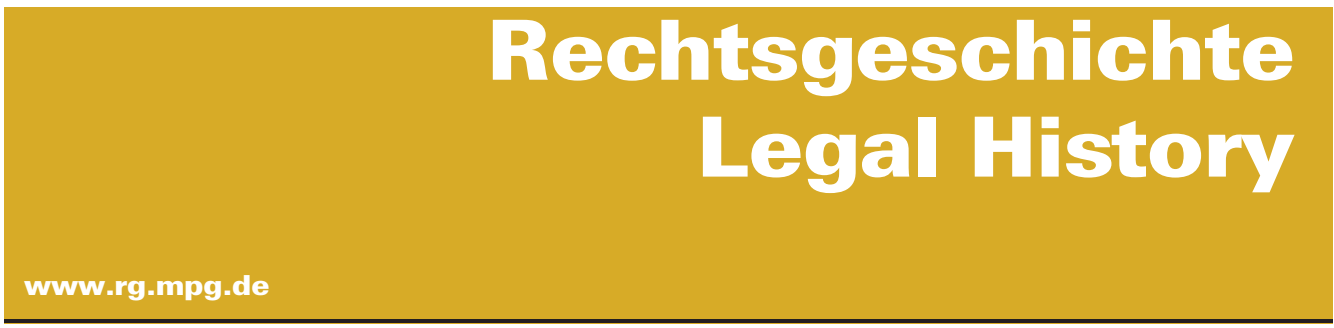

http://www.rg-rechtsgeschichte.de/rg22

$\operatorname{Rg} 22014$

$363-365$

Zitiervorschlag: Rechtsgeschichte - Legal History Rg 22 (2014)

http://dx.doi.org/10.12946/rg22/363-365

\title{
Harald Maihold
}

\section{Ich sündige, also bin ich!}

Das spanische Drama als Probebühne für moralische Experimente 


\section{Harald Maihold}

\section{Ich sündige, also bin ich!}

Das spanische Drama als Probebühne für moralische Experimente*

Literatur- und Rechtsgeschichte haben bisher, jenseits der "Law and Literature «-Bewegung, kaum Berührungspunkte. Dass literarische Quellen aber einen reichen Fundus an moralischen Vorstellungen bereithalten, der eine rechtshistorische Würdigung verdiente, zeigt eine neue theaterwissenschaftliche Studie. Anknüpfend an ihr 2007 erschienenes Buch "Conscience on Stage«, geht Hilaire Kallendorf dem Sündenbegriff in frühneuzeitlichen spanischen Komödien nach, von denen sie, unterstützt durch eine digitale Edition, mehr als 800 Stücke auswertet, ergänzt durch Hinweise auf das englische Renaissancedrama und auf Bildmaterial. Strukturiert, lebendig und oft mit einem Augenzwinkern, ohne ihre Leser mit Zusammenfassungen der Stücke zu langweilen, zeigt die Autorin, in welcher Weise die spanischen Dramen die traditionellen moraltheologischen Organisationskategorien, d.h. die Todsündenlehre und die Zehn Gebote, einem kritischen Transformationsprozess unterwarfen.

Auf die Wurzeln des Theaters als moralischen Spiegels weist Kallendorf immer wieder hin. Doch bemüht sie auch die Marxistische Begriffswelt, wenn sie die Kategorien der Sündenlehre als »komplexe Moralökonomie « verstanden wissen will, in der Vorstellungen gehandelt, getauscht und ersetzt werden (5). Nach einleitenden Bemerkungen über die Methode und das untersuchte Archivmaterial stellt Kallendorf kurz den Forschungsstand dar, wonach die mittelalterliche Lehre von den sieben Todsünden in der frühen Neuzeit zunehmend durch den Rückgriff auf den Dekalog abgelöst worden sei und dies den Übergang von einer kollektivistischen $\mathrm{zu}$ einer individualistischen Sichtweise markiere (6f.). Die Untersuchung gliedert sich in acht Kapitel - und (die bekannte Einteilung Raymond Williams' aufgreifend) in drei Blöcke -, die den sieben Todsünden und den Zehn Geboten gewidmet sind.

Der erste Block (15-94) zeigt, dass Hochmut, Habgier und Wollust in der frühen Neuzeit ihren Stellenwert als moralische Ordnungskategorien weitgehend beibehielten. Kallendorf führt dies darauf zurück, dass wichtige Gebote des Dekalogs diesen Sünden entsprachen: Im Fall des Hochmuts waren es die Verbote von Idolatrie und Blasphemie, im Fall der Habgier, die in den verschiedensten Varianten thematisiert wird, das Diebstahlsverbot und im Fall der Wollust das Verbot des Ehebruchs, die für einen reibungslosen Übergang vom Todsünden- zum Dekalogschema sorgten. Zugleich habe sich als Alternative zu religiösen Sündenlehren im Fall des Ehebruchs ein verstärkt säkularer Bezugsrahmen angeboten.

Im zweiten Block (97-151) werden Differenzierungen angesprochen, die die Todsündenlehre durch die Inkorporation des Dekalogschemas erfuhr: Faulheit, Völlerei und Hass waren im Katalog der Zehn Gebote nicht mehr direkt enthalten (aber indirekt die Faulheit und die Völlerei im Gebot der Feiertagsheiligung mit Fastengebot, der Hass im Tötungsverbot). Sie erwiesen sich deshalb in den Komödien als weniger stabil und erfuhren partiell sogar eine positive Umdeutung: Müßiggang und gutes Essen waren in der höfischen Gesellschaft Spaniens für die Edelleute weniger eine Sünde als vielmehr Pflicht und (auch im Jenseits!) verdienter Lohn, und auch der Zorn wurde als Attribut Gottes nicht mehr geächtet, sondern, innerhalb gerechter Bahnen, als nachahmungswürdig empfunden (diskutiert wurde in den Dramen etwa die Tötung der ehebrecherischen Frau durch ihren Ehemann, die vom weltlichen Recht erlaubt wurde). Indem die Tötung in einen juristischen Kontext gestellt wurde, machte sich gegenüber der

* Hilaire Kallendorf, Sins of the

Fathers. Moral Economies in Early

Modern Spain, Toronto u. a.:

University of Toronto Press 2013.

XIII, 446 S., ISBN 978-1-4426-4458-8 
Sündenlehre auch hier ein verstärkt säkularer Bezugsrahmen bemerkbar; der Zorn verwandelte sich von einer Sünde zur Leidenschaft.

Der dritte Block ist den Brüchen mit der Todsündenlehre gewidmet. Kallendorf zeigt, wie der Neid (155-173), der in den letzten beiden Geboten des Dekalogs fortlebte, in der höfischen Kultur ubiquitär wurde, als Sündenkategorie aber verschwand. Angesichts der spanischen Erfolge in der Neuen Welt bestand Kallendorf zufolge kein Objekt mehr für einen Neid der Spanier außer auf sich selbst. An seine Stelle traten positiv bewertete Eigenschaften wie Ansporn, Mitleid und (National-)Stolz, der insbesondere im niederen Landadel materielle Not kompensieren half. Zwei der zehn Gebote, die im Todsündenkatalog noch keine Entsprechung fanden, traten nach Kallendorf in das entstandene moralische Vakuum (174-201) ein: In der höfischen Kultur eines zunehmend verarmten Spaniens sei die Erziehung zum Gehorsam gegenüber den Eltern ein wichtiges immaterielles Erbe gewesen. Übertragene Bedeutungen des Gebotes hätten durchaus subversive Züge getragen, etwa indem Gott als himmlischer Vater gegenüber den irdischen Eltern ausgespielt wurde oder die Haftung der Söhne für die Sünden der Väter deren schlechtes Beispiel erst offenbarte. Das Verbot des falschen Zeugnisses war in den Dramen Anlass für eine generelle Debatte um Wahrheit und Lüge. Die in der höfischen Gesellschaft allgegenwärtige Intrige verlor ihren Charakter als Sünde, an ihre Stelle traten säkulare Debatten um den Beweis der Wahrheit vor Gericht.

So unterstützt Kallendorfs Studie die These von der Ersetzung der Todsündenlehre durch den Dekalog als moralischer Organisationskategorie in der frühen Neuzeit, zieht allerdings auch die problematische Gleichsetzung des Todsündenschemas mit kollektivistischen und des Dekalogs mit individualistischen Vorstellungen in Zweifel. ${ }^{\mathbf{1}} \mathrm{Im}$ Schlusswort (209-211) wendet sich die Autorin gegen bisherige Deutungen, die Entwicklung einseitig als Siegeszug des Dekalogs oder der Todsündenlehre zu begreifen. Stattdessen betont sie die individualistischen Aspekte beider Schemata. Die
Auseinandersetzung mit den Sünden auf der Bühne, die das Theater zur Probebühne für moralische Experimente gemacht habe, habe in der Spanischen Gesellschaft zu einem Prozess der Selbsterkenntnis und der Subjektivierung geführt. Umfangreiche Endnoten, eine Bibliographie sowie Verzeichnisse runden das Buch ab.

Trotz der interdisziplinären Zusammenhänge der Thematik bleibt Kallendorfs Studie eindimensional auf die spanischen Dramen beschränkt. Künstlerische Überzeichnungen in den Dramen werden von der Autorin durchaus in Rechnung gestellt, ein interdisziplinären Ansprüchen genügender Vergleich der literarischen mit rechts- bzw. theologiehistorischen Quellen wird jedoch nicht einmal angedacht. Die reiche moraltheologische Literatur der frühen Neuzeit, die zur Zeit der untersuchten Komödien von der Spanischen Spätscholastik getragen wird, wird leider nicht unmittelbar berücksichtigt. Auch von der Sekundärliteratur, die sich sowohl mit den moraltheologischen Ordnungskriterien als auch mit der Kategorie der "Vaterstrafe« ausführlich beschäftigt hat, wird nur eine kleine Auswahl, namentlich die Untersuchung Elena del Río Parras zu den spanischen Beichtsummen (Cartografás de la conciencia espanola en la Edad de Oro, 2008), herangezogen. Die großen Namen dieser Zeit - Vitoria, Covarrubias, Soto, Vásquez, Molina und Suárez - werden, wie auch die Sündenspezialisten des Mittelalters Abelard, Gratian, Hostiensis, Panormitanus, Raymundus von Peniaforte - nicht einmal erwähnt, ja sogar Thomas von Aquin taucht nur einmal auf. Das muss als Versäumnis in einer Arbeit verbucht werden, die andererseits neben Michel Foucault, Max Weber und Jacques Derrida auch Gerhard Schröder, Brad Pitt und Speedy Gonzalez einer Erwähnung für wert hält. Denn tragfähige Aussagen über die untersuchten Transformationsprozesse lassen sich eigentlich nur erzielen, wenn die Deutungsmuster der Sünde in den spanischen Dramen mit den Anschauungen der Zuständigen der Zeit, und das waren die Moraltheologen und Kanonisten aus dem Umfeld der Spanischen Spätscholastik, in ein Verhältnis gesetzt werden. So
1 Der Dekalog in Exodus 20 ist mit seinen einleitenden Worten, in denen die Sünden der Väter bis ins dritte und vierte Glied verfolgt werden, keinesfalls Ausdruck einer rein individualistischen Moral, dazu auch
Harald Maihold (2005), Strafe für fremde Schuld? Die Systematisierung des Strafbegriffs in der Spanischen Spätscholastik und Naturrechtslehre, Köln u. a., 277 ff. 
wäre es interessant zu erfahren, ob die von Kallendorf vorgenommenen Verknüpfungen der Todsünden mit den Zehn Geboten auch von den Moraltheologen so gezogen wurden. Der Stellenwert der einzelnen Todsünden hätte näher untersucht werden können (in den Beichtsummen löste die Habgier den Hochmut als schwerste Sünde ab). Auch hätten weitere Ordnungskategorien wie die Sakramente, die vier Kardinaltugenden oder die Werke der Barmherzigkeit, die in den moraltheologischen Werken ebenfalls eine große Rolle spielen, in die Untersuchung aufgenommen werden können. Dass diese Aufgabe von einer einzelnen Unter- suchung wie der vorliegenden nicht geleistet werden kann, versteht sich von selbst und gereicht Kallendorf nicht zum Vorwurf. Doch auf die Perspektiven einer interdisziplinären Weiterführung der Studie hätte hingewiesen werden müssen. Trotz oder gerade wegen dieses Versäumnisses sollte die Beschreibung der Sündenlehre in den spanischen Dramen für unsere Zunft weder Anlass für Neid noch Faulheit sein, sondern zum Ansporn, das dargelegte Material einer rechtshistorischen Würdigung zuzuführen.

\section{Wim Decock}

\section{Papsttreu bis zum Tode*}

Zu der herkömmlichen Vorstellung des Verhältnisses von Kirche und Staat im frühneuzeitlichen Frankreich gehört, dass Fürsten wie Ludwig XIV. die externe Bevormundung der französischen Kirche durch den Bischof von Rom zugunsten der Formierung einer nationalen Sonderkirche endgültig eingedämmt haben. Dementsprechend sind die 1682 unter Leitung von Jacques Bénigne Bossuet verfassten gallikanischen Artikel klassischer Bestandteil des französischen Selbstverständnisses in Sachen Politik und Religion geworden. Sie gelten als das natürliche Ergebnis einer logischen Entwicklung hin zur Verstetigung der Machtansprüche der lokalen weltlichen und geistlichen Machthaber dem Papst gegenüber, die spätestens mit der 1438 durch Karl VII. verabschiedeten Pragmatischen Sanktion von Bourges ihren ersten großen Erfolg feierte. Dass diese Errungenschaft nicht unumkämpft war, belegt nun allerdings die Dissertation von Cyrille Dounot über den äußerst papsttreuen Juristen Antoine Dadine d'Auteserre (1602-1682). Eine reichlich dokumentierte Biographie dieses Zivilrechtlehrers der Universität
Toulouse, die teilweise auf bisher unerforschte Archivmaterialien zurückgeht, bietet der Autor im ersten Teil seiner Arbeit an.

Aufgrund einer gründlichen Analyse vor allem des kanonistischen Schriftums Dadines zeigt der Autor anschließend im zweiten Teil seines Buches auf, was für eine bissige Auseinandersetzung es im Frankreich der zweiten Hälfte des 17. Jahrhunderts zwischen Gallikanern und Ultramontanen gegeben hat. Dadine wurde 1661 von der französischen Bischofskonferenz damit beauftragt, das gallikanische Traktat über den weltlichen Rekurs gegen Missbrauch der kirchlichen Gewalt (l'appel comme d'abus) vom burgundischen Rechtsanwalt Charles Fevret zu widerlegen. Bekanntlich spielten historische Argumente in dieser Auseinandersetzung eine bedeutende Rolle (es sei z. B. auf das 2009 erschienene Heft über den Gallikanismus in der Revue de l'Histoire des Religions verwiesen). Dementsprechend setzte Dadine dem gallikanischen Idealmodell der alten Afrikanischen Kirche das gregorianische Reformmodell entgegen. Er betonte die Superiorität des Römischen Rechts und des päpst-

\footnotetext{
Cyrille Dounot, L'œuvre canonique d'Antoine Dadine d'Auteserre (1602-1682). L'érudition au service de la juridiction ecclésiastique, Toulouse: Presses de l'Université 2013, 757 S, ISBN 978-2-36170-055-3
} 